\title{
GENERALIZED SAMUEL MULTIPLICITIES OF MONOMIAL IDEALS AND VOLUMES
}

\author{
R. ACHILLES AND M. MANARESI
}

\begin{abstract}
We describe conjecturally the generalized Samuel multiplicities $c_{0}, \ldots, c_{d-1}$ of a monomial ideal $I \subset K\left[x_{1}, \ldots, x_{d}\right]$ in terms of its Newton polyhedron $\Gamma(I)$. More precisely, we conjecture that $c_{i}$ equals the sum of the normalized $(d-i)$-volumes of pyramids over the projections of the $(d-i-1)$-dimensional compact faces of $\Gamma(I)$ along the infinite-directions of $i$-unbounded facets in which they are contained. For $c_{0}$ proofs are known (Guibert, Jeffries and Montaño) and for $c_{d-1}$ a proof will be given.
\end{abstract}

\section{INTRODUCTION}

In this paper, based on computations with the free softwares Germenes by A. Montesinos [11] and ReducE [12] by A. C. Hearn [8] and REDUCE developers, we give a conjecture that in the case of monomial ideals links the generalized multiplicities defined algebraically in [3] with volumes derived from the Newton polyhedra of the ideals, thus extending a result of B. Teissier [14].

In 1988, B. Teissier [14, p. 131] proved that for an $\mathfrak{m}$-primary monomial ideal $I$ of a local ring $A$ the Samuel multiplicity is equal to the normalized volume of the complement of the Newton polyhedron of the ideal I. In 1999, G. Guibert [7] generalized Teissier's result. Precisely, Guibert defines the local Segre class of an ideal generated by a set of germs of holomorphic functions and, under a non-degeneracy condition, he describes such a class by Minkowski mixed volumes of polytopes. As a special case he obtains that for a certain class of monomial ideals the local Segre class is a normalized volume of the simplex generated by the origin and the vertices of the Newton polyhedron, see [7, 4.2]. By [4], the local Segre class is the so called $j$-multiplicity of the ideal. In 2013, J. Jeffries and J. Montaño [9] gave a different proof that the

2010 Mathematics Subject Classification. Primary 13H15; Secondary 13F20, $52 \mathrm{~B} 20$.

Key words and phrases. Monomial ideal, generalized Samuel multiplicity, Newton polyhedron. 
$j$-multiplicity of a monomial ideal is the normalized volume of the pyramid of the ideal.

The $j$-multiplicity of an ideal is different from zero if and only if its analytic spread is maximal, that is, equal to the Krull-dimension $d$ of $A$. A result of $\mathrm{C}$. Bivià-Ausina [5] states that the analytic spread diminished by one is the maximum of the dimensions of compact faces of the Newton polyhedron of $I$.

According to [3] the $j$-multiplicity is only the first coordinate of the generalized Samuel multiplicity vector $c(I)=\left(c_{0}(I), \ldots, c_{d}(I)\right)$. Here we present and illustrate a conjecture which expresses the other components of $c(I)$ in terms of the Newton polyhedron of $I$. Our conjecture extends the known result of G. Giubert and J. Jeffries and J. Montaño regarding $c_{0}(I)$, but we shall prove it here only for $c_{d-1}(I)$.

\section{Generalized Samuel multiplicities}

This section is a quick review of a generalization of Samuel's multiplicity by a sequence of numbers, the so-called generalized Samuel multiplicity, which we have introduced in [3].

Let $A$ be a $d$-dimensional Noetherian local ring $(A, \mathfrak{m})$ with unique maximal ideal $\mathfrak{m}$ or a standard graded algebra $A=\bigoplus_{i \geq 0} A_{i}$ such that $A_{0}$ is a field and $\mathfrak{m}=\left(A_{1}\right) A$ is the unique homogeneous maximal ideal of $A$. Let $I \subset A$ be an arbitrary ideal (not necessarily $\mathfrak{m}$-primary).

In order to define the generalized Samuel multiplicity $c(I)$, consider $G_{I}(A):=\bigoplus_{j \geqslant 0} I^{j} / I^{j+1}$, the associated graded ring of $A$ with respect to $I$ and the bigraded ring

$$
T=\bigoplus_{i, j \geq 0} T_{i j}=G_{\mathfrak{m}}\left(G_{I}(A)\right)=\bigoplus_{i, j \geq 0} \frac{\mathfrak{m}^{i} I^{j}+I^{j+1}}{\mathfrak{m}^{i+1} I^{j}+I^{j+1}},
$$

where $T_{00}=A / \mathfrak{m}=K$ is a field.

Let $H^{(0,0)}(i, j):=\operatorname{dim}_{K} T_{i j}$ be the Hilbert function of the bigraded ring $T$ and let

$$
H^{(1,1)}(i, j):=\sum_{q=0}^{j} \sum_{p=0}^{i} H^{(0,0)}(p, q)
$$

its twofold sum transform. For both $i, j \gg 1$ this function becomes a polynomial in $(i, j)$, which can be written in the form

$$
\sum_{k+l \leqslant d} a_{k, l}^{(1,1)}\left(\begin{array}{c}
i+k \\
k
\end{array}\right)\left(\begin{array}{c}
j+l \\
l
\end{array}\right) .
$$


Following [3] define the generalized Samuel multiplicity to be the vector

$$
\begin{aligned}
\left(a_{0, d}^{(1,1)}, a_{1, d-1}^{(1,1)}, \ldots, a_{d, 0}^{(1,1)}\right)=: & \left(c_{0}(T), c_{1}(T), \ldots, c_{d}(T)\right)=: c(T) \\
= & :\left(c_{0}(I), c_{1}(I), \ldots, c_{d}(I)\right)=: c(I) .
\end{aligned}
$$

The first coefficient $c_{0}(I)$ plays an important role as an intersection number and was introduced in [2]. It is called the $j$-multiplicity $j(I):=$ $c_{0}(I)$.

The generalized Samuel multiplicities depend only on the highest dimensional components of $T$, see [15] or [3, Proposition 1.2]:

Proposition 1. With the preceding notation,

$$
c(I)=c(T)=\sum_{P} \operatorname{length}\left(T_{P}\right) \cdot c(T / P),
$$

where $P$ runs through all highest dimensional prime ideals of $T$.

By analogy with the application of $c(I)$ to intersection theory, we shall call $c_{i}(T / P) \neq 0$ a movable contribution to $c_{i}(I)$ if there is an integer $k>i$ such that $c_{k}(T / P) \neq 0$.

\section{A CONJECTURE AND SOME RESUlts}

Let $I$ be an ideal in $R=K\left[x_{1}, \ldots, x_{d}\right]=K[\mathbf{x}]$ ( $K$ a field $)$ minimally generated by the monomials

$$
\mathbf{x}^{v_{1}}:=x_{1}{ }^{v_{1}(1)} \cdots x_{d}^{v_{1}(d)}, \ldots, \mathbf{x}^{v_{r}}:=x_{1}^{v_{r}(1)} \cdots x_{d}^{v_{r}(d)},
$$

that is, $v_{1}=\left(v_{1}(1), \ldots, v_{1}(d)\right), \ldots, v_{r}=\left(v_{r}(1) \ldots v_{r}(d)\right)$ are the points of $\mathbb{Z}_{\geqslant 0}^{d}$ corresponding to the exponents of the generators of $I$.

The Newton polyhedron $\Gamma(I)$ of $I$ is defined as the convex hull of $\left.\left\{v \in \mathbb{Z}_{\geqslant 0}^{d} \mid \mathbf{x}^{v}\right\} \in I\right\}$ in $\mathbb{R}^{d}$, that is,

$$
\begin{aligned}
\Gamma(I) & :=\operatorname{conv}\left(\left\{v \in \mathbb{Z}_{\geqslant 0}^{d} \mid x_{1}^{v(1)} \cdots x_{d}^{v(d)} \in I\right\}\right) \\
& =\operatorname{conv}\left(\left\{v_{1}, \ldots, v_{r}\right\}\right)+\mathbb{R}_{\geqslant 0}^{d},
\end{aligned}
$$

where + denotes the Minkowski sum (for the equality see [13, Lemma 4.3]).

A hyperplane

$$
H=\left\{v \in \mathbb{R}^{d} \mid\langle v, a\rangle=b\right\} \quad\left(\text { with } a \in \mathbb{R}_{\geqslant 0}^{d}, b \in \mathbb{R}\right)
$$

is called a supporting hyperplane of the Newton polyhedron $\Gamma(I)$ if

$$
\Gamma(I) \subset H^{+}=\left\{v \in \mathbb{R}^{d} \mid\langle v, a\rangle \geqslant b\right\} \text { and } \Gamma(I) \cap H \neq \emptyset .
$$

A subset $F \subset \Gamma(I)$ is called a proper face of $\Gamma(I)$ if there exists a supporting hyperplane $H$ of $\Gamma(I)$ such that $F=\Gamma(I) \cap H$. The 
boundary of $\Gamma(I)$ is a set of faces of dimension $d-1$, called facets of $\Gamma(I)$, some of them compact.

The zero-dimensional faces are called vertices of $\Gamma(I)$. We shall denote the set of vertices by $\operatorname{vert}(I)$. Note that the monomials corresponding to the points in $\operatorname{vert}(I)$ are part of the set of minimal generators of $I$, so by renumbering we will assume that

$$
\operatorname{vert}(I)=\left\{v_{1}, \ldots, v_{s}\right\} \text { with some } s \leq r,
$$

hence

$$
\Gamma(I)=\operatorname{conv}\left(\left\{v_{1}, \ldots, v_{r}\right\}\right)+\mathbb{R}_{\geqslant 0}^{d}=\operatorname{conv}\left(\left\{v_{1}, \ldots, v_{s}\right\}\right)+\mathbb{R}_{\geqslant 0}^{d} .
$$

Any face $F$ can be described using its vertices and infinite-directions. Let $e_{j}$ denote the unit vector with non-zero $j$ th component, let $H$ be a supporting hyperplane such that $F=\Gamma(I) \cap H$ and let $a$ be a normal vector to $H$. Then the infinite-directions of $F$ are given by those $e_{j}$ such that the $j$ th component of $a$ is zero. If $v_{i_{1}}, \ldots, v_{i_{s}}$ are the vertices of $F$, then

$$
F=\operatorname{conv}\left(\left\{v_{i_{1}}, \ldots, v_{i_{s}}\right\}\right)+\sum_{j: a(j)=0} \mathbb{R}_{\geq 0} e_{j} .
$$

Of course, the compact faces are precisely those that do not have infinite directions $e_{j}$.

By the Minkowski-Weyl Theorem for convex polyhedra, there are uniquely determined finitely many closed half spaces

$$
H_{i}^{+}=\left\{v \in \mathbb{R}^{d} \mid\left\langle v, a_{i}\right\rangle \geqslant b_{i}\right\}\left(\text { with } a_{i} \in \mathbb{Z}_{\geqslant 0}^{d}, b_{i} \in \mathbb{Z}_{\geqslant 0}\right), i=1, \ldots, t,
$$

such that

$$
\Gamma(I)=H_{1}^{+} \cap \cdots \cap H_{t}^{+} .
$$

Then $F_{i}:=H_{i} \cap \Gamma(I), i=1, \ldots, t$, are the facets of $\Gamma(I)$. We will assume that $H_{1}, \ldots, H_{r}$ are the hyperplanes corresponding to the unbounded facets and that $H_{r+1}, \ldots, H_{t}$ are those corresponding to the compact facets.

To each bounded facet $F=\operatorname{conv}\left(\left\{v_{i 1}, \ldots, v_{i s}\right\}\right)$ of $\Gamma(I)$ we associate the polytope (or pyramid)

$$
\hat{F}:=\operatorname{conv}(0, F)=\operatorname{conv}\left(\left\{0, v_{i 1}, \ldots, v_{i s}\right\}\right)
$$

and denote by $\operatorname{vol}_{d}(\hat{F})$ its $d$-dimensional volume and by

$$
\operatorname{Vol}_{d}(\hat{F}):=d ! \operatorname{vol}_{d}(\hat{F})
$$

its normalized volume. 
A facet $F \subset \Gamma(I)$ is called an $h$-unbounded facet if the normal vector $a$ to its supporting hyperplane has at least $h>0$ coordinates $a(j)$ which are zero, that is, the facet has $h$ infinite-directions $e_{j}$.

Let $\mathcal{F}(k)$ be the set of all $(d-(k+1))$-unbounded facets containing at least one $k$-dimensional compact face $F^{k}, 0 \leq k \leq d-2$. We define $\mathcal{F}(d-1)$ to be the set of all compact or bounded facets of $\Gamma(I)$.

To each couple $\left(F^{k}, F^{d-1}\right)$, with $F^{k}$ a $k$-dimensional compact face and $F^{d-1} \in \mathcal{F}(k)$ containing $F^{k}$, we associate a $(k+1)$-dimensional normalized volume $\operatorname{Vol}\left(F^{k}, F^{d-1}\right)$ in the following way. A normal vector to the facet $F^{d-1}$ lies on at least $d-(k+1)$ coordinate hyperplanes. We project $F^{k}$ on all linear subspaces $\mathbb{R}^{k+1} \subseteq \mathbb{R}^{d}$ obtained by intersecting $d-(k+1)$ of these coordinate hyperplanes, that is, we project $F^{k}$ along all possible choices of $d-(k+1)$ infinite-directions of the facet $F^{d-1}$. We obtain polytopes of dimension at most $k$. We consider only the $k$-dimensional polytopes $\operatorname{pr}_{\mathbb{R}^{k+1}}\left(F^{k}\right) \subset \mathbb{R}^{k+1}$ obtained by the aforementioned projections and set

$$
\hat{F}^{k}:=\operatorname{conv}\left(\left\{0, \operatorname{pr}_{\mathbb{R}^{k+1}}\left(F^{k}\right)\right\}\right),
$$

which has dimension $k$ or $k+1$. The volume associated to the couple $\left(F^{k}, F^{d-1}\right)$ is

$$
\operatorname{Vol}\left(F^{k}, F^{d-1}\right):=\min _{\mathbb{R}^{k+1}} \operatorname{Vol}_{k+1}\left(\hat{F}^{k}\right) .
$$

Conjecture 1. For each $k=0, \ldots, d-1$, the generalized Samuel multiplicity of a monomial ideal $I$ is

$$
c_{d-(k+1)}(I)=\sum_{F^{d-1} \in \mathcal{F}(k)} \min _{F^{k}}\left\{\operatorname{Vol}\left(F^{k}, F^{d-1}\right)\right\},
$$

where the minimum is taken over all compact faces $F^{k}$ of $\Gamma(I)$ that are contained in the facet $F^{d-1}$.

Conjecture 2. Each summand in the formula of Conjecture 1 corresponds, in the sense of Proposition 1, to the contribution of a highest dimensional primary component of $T=G_{\mathfrak{m}}\left(G_{I}(R)\right)$ to $c_{d-(k+1)}(I)$.

In particular, the number of compact facets of $\Gamma(I)$ is equal to the the number of d-dimensional associated prime ideals of $T$ that contain $\mathfrak{m}=\left(x_{1}, \ldots, x_{d}\right) R$.

Note that in general the zero-ideal $\mathfrak{n}$ of $T \cong K\left[x_{1}, \ldots, x_{d}, y_{1}, \ldots, y_{r}\right] / \mathfrak{n}$ is a binomial but not a monomial ideal, see [6].

Our conjectures are confirmed by many examples, but so far we do not have a proof except for Conjecture 1 in the extremal cases $k=0$ and $k=d-1$, as it is stated in the following two theorems. 
Theorem 1 (Jeffries and Montaño, [9]). If $I \subset K\left[x_{1}, \ldots, x_{d}\right]$ is a monomial ideal and $F_{r+1}, \ldots, F_{t}$ are the compact facets of the Newton polyhedron $\Gamma(I)$, then

$$
c_{0}(I)=\sum_{i=r+1}^{t} d ! \operatorname{vol}\left(\hat{F}_{i}\right)=\sum_{i=r+1}^{t} \operatorname{Vol}\left(\hat{F}_{i}\right) .
$$

Theorem 2. Let I be a monomial ideal in $R=K\left[x_{1}, \ldots, x_{d}\right]$ generated by $x_{1}^{v_{1}(1)} \cdots x_{d}^{v_{1}(d)}, \ldots, x_{1}^{v_{r}(1)} \cdots x_{d}^{v_{r}(d)}$ and $m_{j}=\min \left\{v_{1}(j), \ldots, v_{r}(j)\right\}$, $j=1, \ldots, d$. Then

$$
c_{d-1}(I)=m_{1}+\cdots+m_{d}
$$

Proof. By [3, Proposition 2.3], $c_{d-1}(I) \neq 0$ if and only if $\operatorname{dim} R / I=$ $d-1$. If $\operatorname{dim} R / I<d-1$, then none of the variables $x_{j}$ appears in all monomials generating $I$, hence $m_{j}=0$ for all $j$, and the result is true. If $\operatorname{dim} R / I=d-1$, then again by [3, Proposition 2.3],

$$
c_{d-1}(I)=\sum_{P} e\left(I R_{P}\right) \cdot e(R / P),
$$

where $P$ runs through all $(d-1)$-dimensional associated prime ideals of $R / I$, that is, prime ideals of the form $\left(x_{j}\right)$ for some $j$, see [10, Satz 9]. Therefore $I R_{P}=\left(x_{j}^{m_{j}}\right) R_{P}$ and $e\left(I R_{P}\right)=m_{j}$. By [10] the $(d-1)$ dimensional part of the primary decomposition of $I$ is $\left(x_{1}^{m_{1}}\right) \cap\left(x_{2}^{m_{2}}\right) \cap$ $\cdots \cap\left(x_{d}^{m_{d}}\right)$, which is of degree $m_{1}+\cdots+m_{d}$.

The following corollary of Theorem 2 states that the Conjecture 1 is true for $k=0$.

Corollary 3. Using the preceding notations, for $j=1, \ldots, d$ set

$$
F_{j}:=\operatorname{conv}\left(\left\{v \in \operatorname{vert}(I) \mid v(j)=m_{j}\right\}\right)+\sum_{1 \leq i \leq d, i \neq j} \mathbb{R}_{\geqslant 0} e_{i}
$$

and $\operatorname{vert}\left(F_{j}\right):=\operatorname{vert}(I) \cap F_{j}$.

Then $\mathcal{F}(0)=\left\{F_{1}, \ldots, F_{d}\right\}$. If $v \in \operatorname{vert}\left(F_{j}\right)$, then $\operatorname{Vol}\left(v, F_{j}\right)=m_{j}$ and it holds

$$
c_{d-1}(I)=\sum_{j=1}^{d} \min _{v \in \operatorname{vert}\left(F_{j}\right)}\left\{\operatorname{Vol}\left(v, F_{j}\right)\right\} .
$$

Proof. Since each $v \in \Gamma(I)$ is the sum of a convex combination of the vertices $v_{1}, \ldots, v_{s}$ of $\Gamma(I)$ and of some $w \in \mathbb{R}_{\geqslant 0}^{d}$, we have

$$
v(j) \geq \min \left\{v_{1}(j), \ldots, v_{s}(j)\right\}+w(j) \geq \min \left\{v_{1}(j), \ldots, v_{s}(j)\right\},
$$


hence

$$
m_{j}:=\min \left\{v_{1}(j), \ldots, v_{r}(j)\right\}=\min \left\{v_{1}(j), \ldots, v_{s}(j)\right\}=\min _{v \in \Gamma(I)}\{v(j)\} .
$$

It follows that $F_{1}, \ldots, F_{d}$ are precisely the $(d-1)$-unbounded facets of $\Gamma(I)$, that is, $\mathcal{F}(0)=\left\{F_{1}, \ldots, F_{d}\right\}$.

Since the projection of $\mathbb{R}^{d} \rightarrow \mathbb{R}$ with center $\sum_{1 \leq i \leq d, i \neq j} \mathbb{R} e_{i}$ sends $v \in \operatorname{vert}\left(F_{j}\right)$ to $v(j)=m_{j}$, we have $\operatorname{Vol}\left(v, F_{j}\right)=v(j)=m_{j}$. Then, by Theorem 2, we obtain the desired formula for $c_{d-1}$, which finishes the proof.

\section{EXAMPLES}

We illustrate the theorems and the conjecture by examples of monomial ideals $I \subset R=K\left[x_{1}, \ldots, x_{d}\right], K$ an arbitrary field. We set $\mathfrak{m}:=\left(x_{1}, \ldots, x_{d}\right) R$ and $T:=G_{\mathfrak{m}}\left(G_{I}(R)\right)$. All the examples will show a closed relation between the summands in the formula of Conjecture 1 and the highest dimensional primary components of $T$.

We begin with the simplest case of a monomial ideal generated by one monomial in two variables. The first two examples are covered by Theorems 1 and 2.

Example 1 (Figure 1). Let $I=\left(x^{3} y^{2}\right) \subset R=K[x, y]$. We have

$$
c(I)=\left(c_{0}(I), c_{1}(I), c_{2}(I)\right)=(0,5,0)=2 \cdot(0,1,0)+3 \cdot(0,1,0),
$$

where the summands are the contributions of the components of the bigraded ring $G_{\mathfrak{m}}\left(G_{I}(R)\right)$, see Proposition 1 .

The Newton polyhedron $\Gamma(I)$ has only one vertex $v=(3,2)$ and two (unbounded) facets $F_{1}=v+R_{\geq 0} e_{2}$ and $F_{2}=v+R_{\geq 0} e_{1}$ (see Figure 1), hence $\mathcal{F}(1)=\emptyset$ and $c_{0}(I)=0$. We have $\mathcal{F}(0)=\left\{F_{1}, F_{2}\right\}$ and $\operatorname{Vol}\left(v, F_{1}\right)=3$ and $\operatorname{Vol}\left(v, F_{2}\right)=2$, hence $c_{1}(I)=5$.

Example 2 (Figure 2). Let $I=\left(x^{2} y^{5}, x^{3} y^{4}, x^{4} y^{2}, x^{6} y\right) \subset R=K[x, y]$.

We have

$$
\begin{aligned}
c(I) & =\left(c_{0}(I), c_{1}(I), c_{2}(I)\right) \\
& =(24,3,0)=16 \cdot(1,0,0)+8 \cdot(1,0,0)+2 \cdot(0,1,0)+(0,1,0),
\end{aligned}
$$

where the summands are the contributions of the components of the bigraded ring $G_{\mathfrak{m}}\left(G_{I}(R)\right)$, see Proposition 1 .

The Newton polyhedron $\Gamma(I)$ has three vertices $v_{1}=(2,5), v_{3}=$ $(4,2), v_{4}=(6,1)$, two (unbounded) facets $F_{1}=v_{1}+R_{\geq 0} e_{2}, F_{2}=v_{4}+$ 


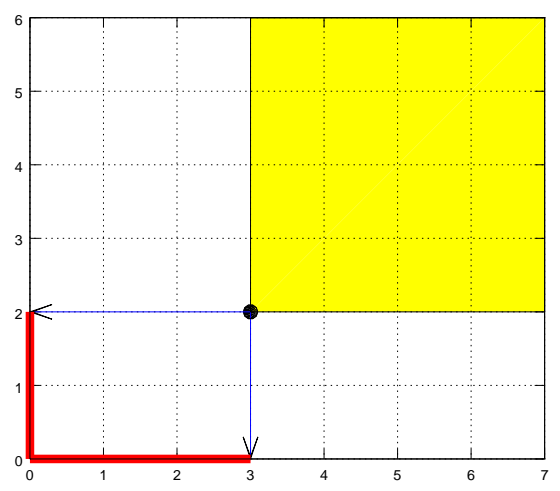

FiguRE 1. Projection along the infinite-directions of the facets gives $c_{1}(I)$, which is the red distance.
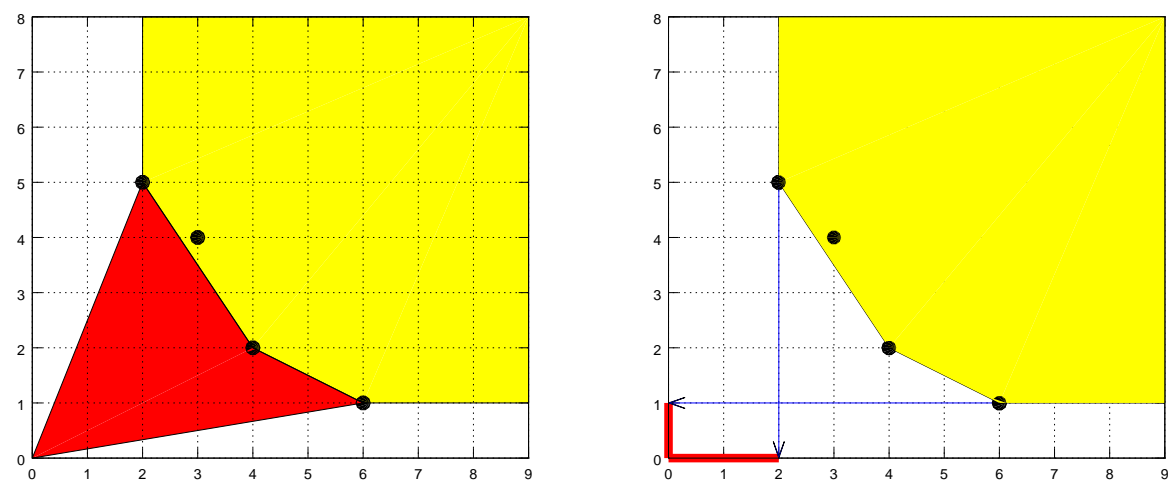

Figure 2. The red area is $c_{0}(I) / 2$, the red distance $c_{1}(I)$.

$R_{\geq 0} e_{1}$ and two bounded facets: the line segments $F_{3}=\operatorname{conv}\left(v_{1}, v_{3}\right), F_{4}=$ $\operatorname{conv}\left(v_{3}, v_{4}\right)$ (see Figure 2), hence $\mathcal{F}(1)=\left\{F_{3}, F_{4}\right\}$ and $c_{0}(I)=\operatorname{Vol}\left(\operatorname{conv}\left(0, F_{3}\right)\right)+\operatorname{Vol}\left(\operatorname{conv}\left(0, F_{4}\right)\right)=\left|\begin{array}{ll}4 & 2 \\ 2 & 5\end{array}\right|+\left|\begin{array}{ll}6 & 1 \\ 4 & 2\end{array}\right|=16+8$.

We have $\mathcal{F}(0)=\left\{F_{1}, F_{2}\right\}$ and

$$
c_{1}(I)=\operatorname{Vol}\left(v_{1}, F_{1}\right)+\operatorname{Vol}\left(v_{4}, F_{2}\right)=2+1=3 .
$$

In the following example some of the compact faces of $\Gamma(I)$ do not contribute to the generalized Samuel multiplicity $c(I)$. In this example there is also a movable contribution, therefore the number of the highest dimensional components of $T$ is one less than the number of summands in the conjectured formula. 

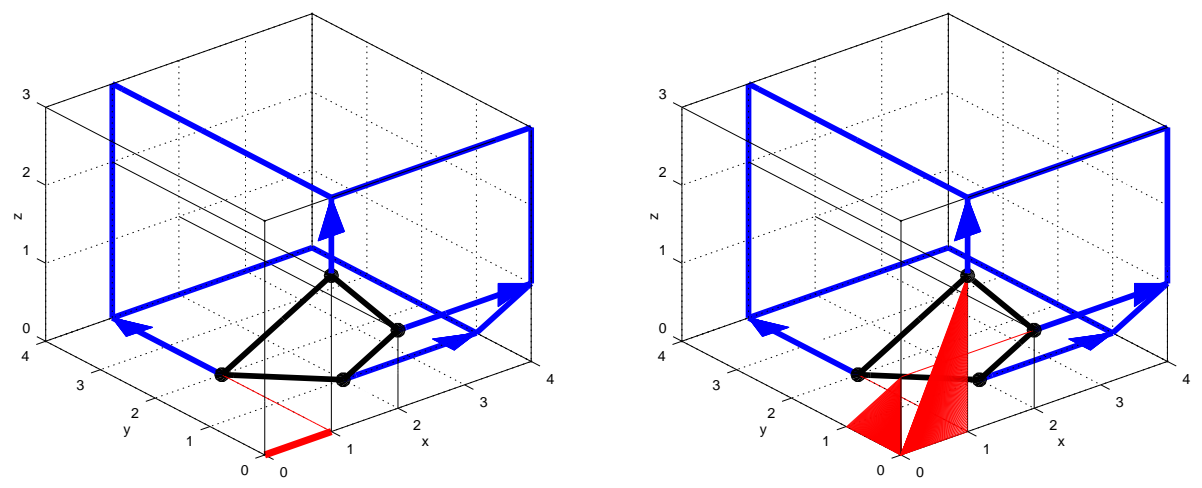

FiguRE 3. Infinite-directions (blue arrows) of the unbounded facets, $c_{2}(I)$ (red distance) and $c_{1}(I) / 2$ (red area).

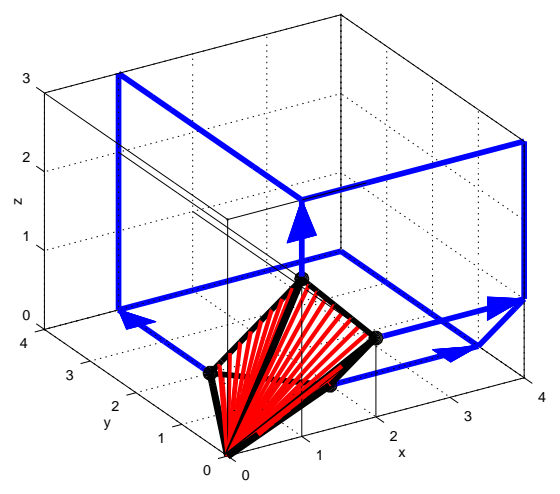

Figure 4 . The volume of the red pyramid is $c_{0} / 6$.

Example 3 (Figures 3, 4 and 5). Let $I=\left(x^{2} y, x^{2} z, x y^{2}, x z^{2}\right) \subset$ $K[x, y, z]$. By a computer computation (using [1]) we have

$$
\begin{aligned}
c(I) & =\left(c_{0}(I), c_{1}(I), c_{2}(I), c_{3}(I)\right) \\
& =(9,3,1,0)=3 \cdot(3,0,0,0)+(0,1,0,0)+(0,2,1,0),
\end{aligned}
$$

where the summands are the contributions of the highest dimensional components of the bigraded ring $T$, see Proposition 1. The contribution 2 in the last vector is a movable contribution to $c_{1}(I)$. This can be read off also from the Newton polyhedron $\Gamma(I)$, see Figure 6 .

According to the program Germenes [11], the compact faces of $\Gamma(I)$ are the vertices $v_{1}=(2,1,0), v_{2}=(2,0,1), v_{3}=(1,2,0), v_{4}=(1,0,2)$, the line segments $v_{1} v_{2}, v_{1} v_{3}, v_{2} v_{4}, v_{3} v_{4}$ and the quadrilateral facet 

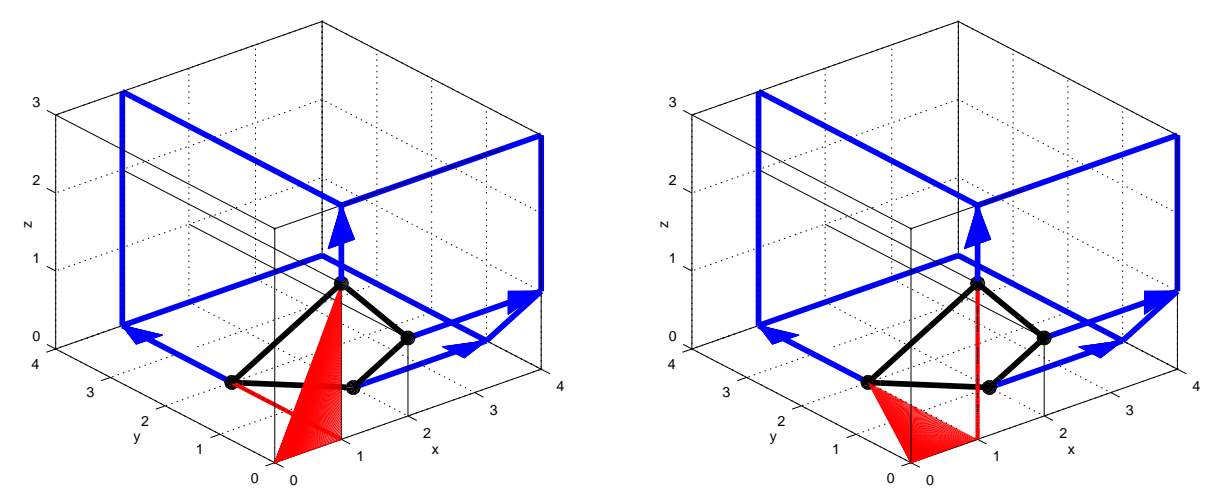

Figure 5. A movable contribution ( to $c_{1}(I) / 2$, red area) can be realized by at least two projections.

$v_{1} v_{2} v_{4} v_{3}$. The unbounded facets are

$$
\begin{aligned}
& F_{1}=v_{3} v_{4}+\mathbb{R}_{\geq 0} e_{2}+\mathbb{R}_{\geq 0} e_{3}, \quad F_{2}=v_{2} v_{4}+\mathbb{R}_{\geq 0} e_{1}+\mathbb{R}_{\geq 0} e_{3}, \\
& F_{3}=v_{1} v_{3}+\mathbb{R}_{\geq 0} e_{1}+\mathbb{R}_{\geq 0} e_{2}, \quad F_{4}=v_{1} v_{2}+\mathbb{R}_{\geq 0} e_{1} .
\end{aligned}
$$

We observe that the set of bounded facets is $\mathcal{F}(2)=\left\{v_{1} v_{2} v_{4} v_{3}\right\}$ and $c_{0}(I)=\operatorname{Vol}\left(\operatorname{conv}\left(0, v_{1}, v_{2}, v_{4}, v_{3}\right)\right)=\left|\begin{array}{lll}2 & 1 & 0 \\ 1 & 2 & 0 \\ 2 & 0 & 1\end{array}\right|+\left|\begin{array}{ccc}2 & 1 & 0 \\ 1 & 2 & 0 \\ 1 & 0 & 2\end{array}\right|=3+6=9$ see Figure 4.

The set of 1-unbounded facets that contain a compact one-dimensional face is $\mathcal{F}(1)=\left\{F_{1}, F_{2}, F_{3}, F_{4}\right\}$, and we have

$$
\begin{gathered}
\operatorname{Vol}\left(v_{1} v_{2}, F_{4}\right)=\left|\begin{array}{ll}
1 & 0 \\
0 & 1
\end{array}\right|=1 \\
\operatorname{Vol}\left(v_{1} v_{3}, F_{3}\right)=\min \left\{\left|\begin{array}{ll}
1 & 0 \\
2 & 0
\end{array}\right|,\left|\begin{array}{ll}
2 & 0 \\
1 & 0
\end{array}\right|\right\}=0, \\
\operatorname{Vol}\left(v_{2} v_{4}, F_{2}\right)=\min \left\{\left|\begin{array}{ll}
0 & 1 \\
0 & 2
\end{array}\right|,\left|\begin{array}{ll}
2 & 0 \\
1 & 0
\end{array}\right|\right\}=0, \\
\operatorname{Vol}\left(v_{3} v_{4}, F_{1}\right)=\min \left\{\left|\begin{array}{ll}
1 & 0 \\
1 & 2
\end{array}\right|,\left|\begin{array}{ll}
1 & 0 \\
1 & 2
\end{array}\right|\right\}=2
\end{gathered}
$$



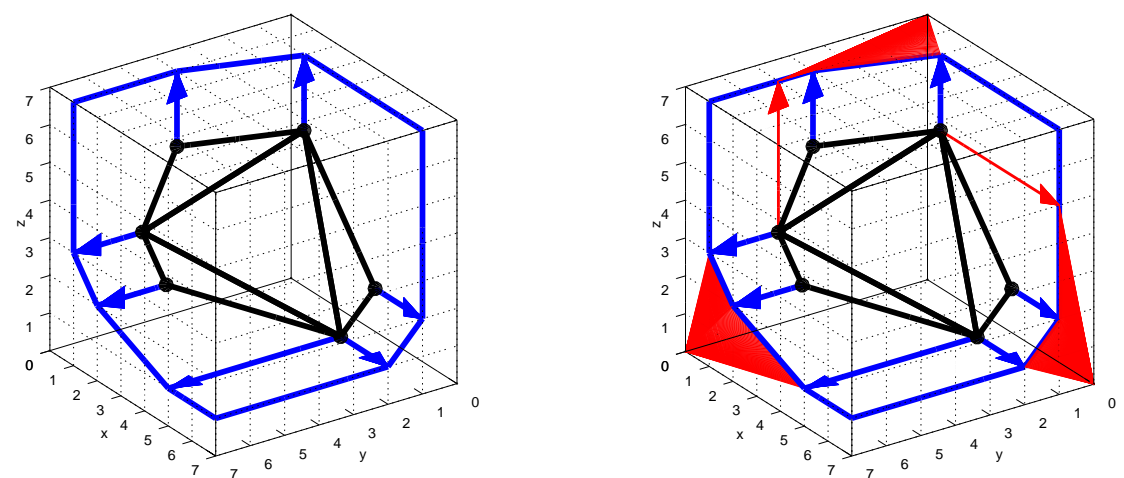

FiguRE 6. $\Gamma(I)$ with compact (black) and unbounded (blue) facets; projections of compact edges along infinitedirections (blue arrows) give $c_{1}(I) / 2$ (red area).

(the last minimum is given by two different projections and is a movable contribution, see Figure 5), hence

$$
\begin{aligned}
c_{1}(I) & =\operatorname{Vol}\left(v_{1} v_{2}, F_{4}\right)+\operatorname{Vol}\left(v_{1} v_{3}, F_{3}\right)+\operatorname{Vol}\left(v_{2} v_{4}, F_{2}\right)+\operatorname{Vol}\left(v_{3} v_{4}, F_{1}\right) \\
& =1+0+0+2=3 .
\end{aligned}
$$

The set of 2-unbounded facets is $\mathcal{F}(0)=\left\{F_{1}, F_{2}, F_{3}\right\}$. We have $\operatorname{Vol}\left(v_{3}, F_{1}\right)=1, \operatorname{Vol}\left(v_{4}, F_{1}\right)=1, \operatorname{Vol}\left(v_{4}, F_{2}\right)=0, \operatorname{Vol}\left(v_{1}, F_{3}\right)=0$, $\operatorname{Vol}\left(v_{3}, F_{3}\right)=0$, hence

$$
\begin{aligned}
c_{2}(I)= & \min \left\{\operatorname{Vol}\left(v_{3}, F_{1}\right), \operatorname{Vol}\left(v_{4}, F_{1}\right)\right\}+\operatorname{Vol}\left(v_{4}, F_{2}\right)+ \\
& +\min \left\{\operatorname{Vol}\left(v_{1}, F_{3}\right), \operatorname{Vol}\left(v_{3}, F_{3}\right)\right\}=1+0+0=1 .
\end{aligned}
$$

Example 4 (Figure 6). Let $I=\left(x y^{4} z^{5}, x^{2} y^{5} z^{2}, x y^{5} z^{3}, x^{5} y z^{2}, x^{2} y z^{5}\right.$, $\left.x^{5} y^{2} z\right) \subset K[x, y, z]$. By a computer computation we have

$$
\begin{aligned}
c(I)= & \left(c_{0}(I), c_{1}(I), c_{2}(I), c_{3}(I)\right)=(168,26,3,0)= \\
= & 19 \cdot(1,0,0,0)+103 \cdot(1,0,0,0)+22 \cdot(1,0,0,0)+ \\
& +24 \cdot(1,0,0,0)+7 \cdot(0,1,0,0)+(0,3,1,0)+ \\
& +8 \cdot(0,1,0,0)+(0,1,0,0)+4 \cdot(0,1,0,0)+ \\
& +3 \cdot(0,1,0,0)+(0,0,1,0)+(0,0,1,0),
\end{aligned}
$$

where the summands are the contributions of the highest dimensional components of the bigraded ring $T$, see Proposition 1 . The contribution 3 in the sixth vector is a movable contribution to $c_{1}(I)$.

The software Germenes [11] gives the following description of the Newton polyhedron $\Gamma(I)$. The compact faces of $\Gamma(I)$ are the 6 vertices 
$v_{1}=(1,4,5), v_{2}=(2,5,2), v_{3}=(1,5,3), v_{4}=(5,1,2), v_{5}=(2,1,5)$, $v_{6}=(5,2,1)$, the 9 line segments $v_{4} v_{6}, v_{2} v_{6}, v_{5} v_{6}, v_{4} v_{5}, v_{3} v_{6}, v_{3} v_{2}, v_{3} v_{5}$, $v_{1} v_{5}, v_{1} v_{3}$ and the 4 triangles (bounded facets) $v_{4} v_{5} v_{6}, v_{2} v_{3} v_{6}, v_{3} v_{5} v_{6}$, $v_{1} v_{3} v_{5}$. There are 7 unbounded facets:

$$
\begin{aligned}
& F_{1}=v_{1} v_{3}+\mathbb{R}_{\geq 0} e_{2}+\mathbb{R}_{\geq 0} e_{3}, \quad F_{2}=v_{4} v_{5}+\mathbb{R}_{\geq 0} e_{1}+\mathbb{R}_{\geq 0} e_{3}, \\
& F_{3}=v_{6}+\mathbb{R}_{\geq 0} e_{1}+\mathbb{R}_{\geq 0} e_{2}, \quad F_{4}=v_{1} v_{5}+\mathbb{R}_{\geq 0} e_{3}, \\
& F_{5}=v_{2} v_{3}+\mathbb{R}_{\geq 0} e_{2}, \quad F_{6}=v_{2} v_{6}+\mathbb{R}_{\geq 0} e_{2}, \quad F_{7}=v_{4} v_{6}+\mathbb{R}_{\geq 0} e_{1} .
\end{aligned}
$$

From the set of bounded facets $\mathcal{F}(2)=\left\{v_{4} v_{5} v_{6}, v_{2} v_{3} v_{6}, v_{3} v_{5} v_{6}, v_{1} v_{3} v_{5}\right\}$ we get

$$
\begin{aligned}
c_{0}(I)= & \operatorname{Vol}\left(\operatorname{conv}\left(0, v_{4}, v_{5}, v_{6}\right)\right)+\operatorname{Vol}\left(\operatorname{conv}\left(0, v_{2}, v_{3}, v_{6}\right)+\right. \\
& +\operatorname{Vol}\left(\operatorname{conv}\left(0, v_{3}, v_{5}, v_{6}\right)+\operatorname{Vol}\left(\operatorname{conv}\left(0, v_{1}, v_{3}, v_{5}\right)=\right.\right. \\
= & \left|\begin{array}{lll}
5 & 1 & 2 \\
5 & 2 & 1 \\
2 & 1 & 5
\end{array}\right|+\left|\begin{array}{lll}
2 & 5 & 2 \\
1 & 5 & 3 \\
5 & 2 & 1
\end{array}\right|+\left|\begin{array}{lll}
1 & 5 & 3 \\
2 & 1 & 5 \\
5 & 2 & 1
\end{array}\right|+\left|\begin{array}{lll}
1 & 4 & 5 \\
2 & 1 & 5 \\
1 & 5 & 3
\end{array}\right|= \\
= & 24+22+103+19=168 .
\end{aligned}
$$

We have $\mathcal{F}(1)=\left\{F_{1}, F_{2}, F_{4}, F_{5}, F_{6}, F_{7}\right\}$ and

$$
\begin{aligned}
& \operatorname{Vol}\left(v_{1} v_{3}, F_{1}\right)=\min \left\{\left|\begin{array}{ll}
1 & 4 \\
1 & 5
\end{array}\right|,\left|\begin{array}{ll}
1 & 3 \\
1 & 5
\end{array}\right|\right\}=\min \{1,2\}=1, \\
& \operatorname{Vol}\left(v_{4} v_{5}, F_{2}\right)=\min \left\{\left|\begin{array}{ll}
5 & 1 \\
2 & 1
\end{array}\right|,\left|\begin{array}{ll}
1 & 2 \\
1 & 5
\end{array}\right|\right\}=\min \{3,3\}=3
\end{aligned}
$$

(here the minimum is attained twice, that is, by two different projections which indicates a movable contribution),

$$
\begin{aligned}
& \operatorname{Vol}\left(v_{1} v_{5}, F_{4}\right)=\left|\begin{array}{ll}
2 & 1 \\
1 & 4
\end{array}\right|=7, \quad \operatorname{Vol}\left(v_{2} v_{3}, F_{5}\right)=\left|\begin{array}{ll}
2 & 2 \\
1 & 3
\end{array}\right|=4, \\
& \operatorname{Vol}\left(v_{2} v_{6}, F_{6}\right)=\left|\begin{array}{ll}
5 & 1 \\
2 & 2
\end{array}\right|=8, \quad \operatorname{Vol}\left(v_{4} v_{6}, F_{7}\right)=\left|\begin{array}{ll}
2 & 1 \\
1 & 2
\end{array}\right|=3,
\end{aligned}
$$

hence

$$
\begin{aligned}
c_{1}(I)= & \operatorname{Vol}\left(v_{1} v_{3}, F_{1}\right)+\operatorname{Vol}\left(v_{4} v_{5}, F_{2}\right)+\operatorname{Vol}\left(v_{1} v_{5}, F_{4}\right)+ \\
& +\operatorname{Vol}\left(v_{2} v_{3}, F_{5}\right)+\operatorname{Vol}\left(v_{2} v_{6}, F_{6}\right)+\operatorname{Vol}\left(v_{4} v_{6}, F_{7}\right)= \\
= & 1+3+7+4+8+3=26 .
\end{aligned}
$$

We observe that the compact 1-dimensional faces $v_{5} v_{6}, v_{3} v_{6}, v_{3} v_{5}$, that is, the edges of the big triangle $v_{3} v_{5} v_{6}$, do not contribute to $c_{1}(I)$ 


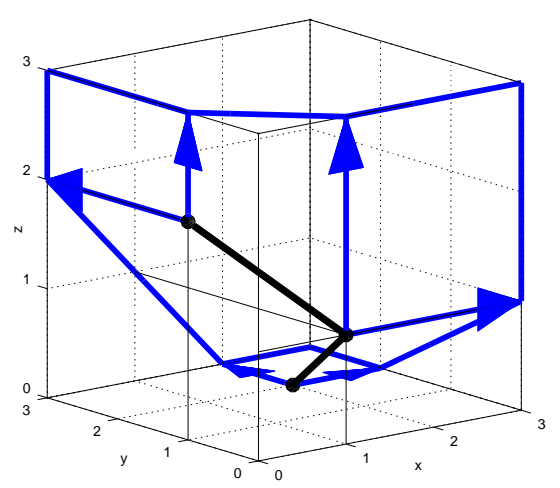

Figure 7 . The triangle defined by 3 affinely independent vertices is not a compact facet.

since they lie on no 1-unbounded facets. Moreover, as in the previous example, there is a movable contribution, namely $\operatorname{Vol}\left(v_{4} v_{5}, F_{2}\right)=3$.

The set of 2-unbounded facets is $\mathcal{F}(0)=\left\{F_{1}, F_{2}, F_{3}\right\}$, and we have $\operatorname{Vol}\left(v_{1}, F_{1}\right)=1, \operatorname{Vol}\left(v_{3}, F_{1}\right)=1, \operatorname{Vol}\left(v_{4}, F_{2}\right)=1, \operatorname{Vol}\left(v_{5}, F_{2}\right)=1$, $\operatorname{Vol}\left(v_{6}, F_{3}\right)=1$, hence

$$
\begin{aligned}
c_{2}(I)= & \min \left\{\operatorname{Vol}\left(v_{1}, F_{1}\right), \operatorname{Vol}\left(v_{1}, F_{1}\right)\right\}+\min \left\{\operatorname{Vol}\left(v_{4}, F_{2}\right), \operatorname{Vol}\left(v_{5}, F_{2}\right)\right\}+ \\
& +\operatorname{Vol}\left(v_{6}, F_{3}\right)=1+1+1=3 .
\end{aligned}
$$

Example 5 (Figure 7). Let $I=\left(x z, x^{2} y^{2}, y z^{2}\right) \subset K[x, y, z]$. Вy a computer computation we have

$$
\begin{aligned}
c(I) & =\left(c_{0}(I), c_{1}(I), c_{2}(I), c_{3}(I)\right)=(0,7,0,0)= \\
& =2 \cdot(0,1,0,0)+2 \cdot(0,1,0,0)+2 \cdot(0,1,0,0)+(0,1,0,0),
\end{aligned}
$$

where the summands are the contributions of the highest dimensional components of the bigraded ring $T$, see Proposition 1.

A computation with the program Germenes [11] shows the the compact faces of $\Gamma(I)$ are the vertices $v_{1}=(1,0,1), v_{2}=(2,2,0), v_{3}=$ $(0,1,2)$ and the line segments $v_{1} v_{2}, v_{1} v_{3}$. There are no compact or bounded facets, but 6 unbounded facets:

$$
\begin{aligned}
& F_{1}=v_{3}+\mathbb{R}_{\geq 0} e_{2}+\mathbb{R}_{\geq 0} e_{3}, \quad F_{2}=v_{1}+\mathbb{R}_{\geq 0} e_{1}+\mathbb{R}_{\geq 0} e_{3}, \\
& F_{3}=v_{2}+\mathbb{R}_{\geq 0} e_{1}+\mathbb{R}_{\geq 0} e_{2}, \quad F_{4}=v_{1} v_{2}+\mathbb{R}_{\geq 0} e_{1}, \\
& F_{5}=v_{1} v_{3}+\mathbb{R}_{\geq 0} e_{3}, \quad F_{6}=v_{1} v_{2} v_{3}+\mathbb{R}_{\geq 0} e_{2} .
\end{aligned}
$$


We observe that $\mathcal{F}(2)=\emptyset$, hence

$$
c_{0}(I)=0 \neq \operatorname{Vol}_{3}\left(\operatorname{conv}\left(\left\{0, v_{1}, v_{2}, v_{3}\right\}\right)\right)=\left|\begin{array}{lll}
1 & 0 & 1 \\
2 & 2 & 0 \\
0 & 1 & 2
\end{array}\right|=6 .
$$

This means that $v_{1}, v_{2}, v_{3}$ are affinely independent, but the local Segre class is zero and not equal to the normalized volume of the simplex generated by the origin and $v_{1}, v_{2}, v_{3}$ as claimed in $[7,4.2]$.

The set of 1-unbounded facets which contain a compact 1-dimensional face is $\mathcal{F}(1)=\left\{F_{4}, F_{5}, F_{6}\right\}$, and we have

$$
\begin{aligned}
& \operatorname{Vol}\left(v_{1} v_{2}, F_{4}\right)=\left|\begin{array}{ll}
2 & 0 \\
0 & 1
\end{array}\right|=2, \quad \operatorname{Vol}\left(v_{1} v_{2}, F_{6}\right)=\left|\begin{array}{ll}
2 & 0 \\
1 & 1
\end{array}\right|=2, \\
& \operatorname{Vol}\left(v_{1} v_{3}, F_{5}\right)=\left|\begin{array}{ll}
1 & 1 \\
0 & 2
\end{array}\right|=2, \quad \operatorname{Vol}\left(v_{1} v_{3}, F_{6}\right)=\left|\begin{array}{ll}
1 & 0 \\
0 & 1
\end{array}\right|=1,
\end{aligned}
$$

hence

$$
\begin{aligned}
c_{1}(I) & =\operatorname{Vol}\left(v_{1} v_{2}, F_{4}\right)+\operatorname{Vol}\left(v_{1} v_{2}, F_{6}\right)+\operatorname{Vol}\left(v_{1} v_{3}, F_{5}\right)+\operatorname{Vol}\left(v_{1} v_{3}, F_{6}\right) \\
& =2+2+2+1=7 .
\end{aligned}
$$

The set of 2-unbounded facets is $\mathcal{F}(0)=\left\{F_{1}, F_{2}, F_{3}\right\}$ and we have

$$
\operatorname{Vol}\left(v_{3}, F_{1}\right)=0, \quad \operatorname{Vol}\left(v_{1}, F_{2}\right)=0, \operatorname{Vol}\left(v_{2}, F_{3}\right)=0,
$$

hence $c_{2}(I)=0$.

In the following example there is only one 1-dimensional compact face, but it lies on three 2-unbounded facets.

Example 6. Let $d=4, I=\left(x_{1}^{3} x_{2} x_{3} x_{4}, x_{1} x_{2} x_{3} x_{4}^{2}\right)$. By a computer computation we have

$$
\begin{aligned}
c(I)= & \left(c_{0}(I), c_{1}(I), c_{2}(I), c_{3}(I), c_{4}(I)\right)=(0,0,7,4,0)= \\
= & 5 \cdot(0,0,1,0,0)+(0,0,1,0,0)+(0,0,1,0,0)+(0,0,0,1,0)+ \\
& +(0,0,0,1,0)+(0,0,0,1,0)+(0,0,0,1,0),
\end{aligned}
$$

where the summands are the contributions of the highest dimensional components of the bigraded ring $T$, see Proposition 1.

The compact faces of the Newton polyhedron $\Gamma(I)$ are the vertices $v_{1}=(3,1,1,1), v_{2}=(1,1,1,2)$ and the line segment $v_{1} v_{2}$. There are 
no compact facets, but 5 unbounded facets:

$$
\begin{aligned}
& F_{1}=v_{2}+\mathbb{R}_{\geq 0} e_{2}+\mathbb{R}_{\geq 0} e_{3}+\mathbb{R}_{\geq 0} e_{4}, \\
& F_{2}=v_{1} v_{2}+\mathbb{R}_{\geq 0} e_{1}+\mathbb{R}_{\geq 0} e_{3}+\mathbb{R}_{\geq 0} e_{4}, \\
& F_{3}=v_{1} v_{2}+\mathbb{R}_{\geq 0} e_{1}+\mathbb{R}_{\geq 0} e_{2}+\mathbb{R}_{\geq 0} e_{4}, \\
& F_{4}=v_{1}+\mathbb{R}_{\geq 0} e_{1}+\mathbb{R}_{\geq 0} e_{2}+\mathbb{R}_{\geq 0} e_{3}, \\
& F_{5}=v_{1} v_{2}+\mathbb{R}_{\geq 0} e_{2}+\mathbb{R}_{\geq 0} e_{3} .
\end{aligned}
$$

Obviously $\mathcal{F}(3)=\mathcal{F}(2)=\emptyset$, hence $c_{0}(I)=c_{1}(I)=0$. We have $\mathcal{F}(1)=\left\{F_{2}, F_{3}, F_{5}\right\}$ and

$$
\begin{gathered}
\operatorname{Vol}\left(v_{1} v_{2}, F_{2}\right)=\min \left\{\left|\begin{array}{ll}
3 & 1 \\
1 & 1
\end{array}\right|,\left|\begin{array}{ll}
1 & 1 \\
1 & 2
\end{array}\right|\right\}=\min \{2,1\}=1, \\
\operatorname{Vol}\left(v_{1} v_{2}, F_{3}\right)=\min \left\{\left|\begin{array}{ll}
3 & 1 \\
1 & 1
\end{array}\right|,\left|\begin{array}{ll}
1 & 1 \\
1 & 2
\end{array}\right|\right\}=\min \{2,1\}=1, \\
\operatorname{Vol}\left(v_{1} v_{2}, F_{5}\right)=\left|\begin{array}{ll}
3 & 1 \\
1 & 2
\end{array}\right|=5,
\end{gathered}
$$

We observe that in the computations of $\operatorname{Vol}\left(v_{1} v_{2}, F_{2}\right)$ and $\operatorname{Vol}\left(v_{1} v_{2}, F_{3}\right)$ the projection of the line segment $v_{1} v_{2}$ on the $\left\{x_{2}, x_{3}\right\}$-plane gives the point $(1,1)$ and must not be considered. We obtain $c_{2}(I)=$ $\operatorname{Vol}\left(v_{1} v_{2}, F_{2}\right)+\operatorname{Vol}\left(v_{1} v_{2}, F_{3}\right)+\operatorname{Vol}\left(v_{1} v_{2}, F_{5}\right)=1+1+5=7$.

The set of 3 -unbounded facets is $\mathcal{F}(0)=\left\{F_{1}, F_{2}, F_{3}, F_{4}\right\}$, and we have

$$
\begin{aligned}
& \operatorname{Vol}\left(v_{2}, F_{1}\right)=1, \quad \operatorname{Vol}\left(v_{1}, F_{2}\right)=1, \quad \operatorname{Vol}\left(v_{2}, F_{2}\right)=1, \\
& \operatorname{Vol}\left(v_{1}, F_{3}\right)=1, \quad \operatorname{Vol}\left(v_{2}, F_{3}\right)=1, \quad \operatorname{Vol}\left(v_{1}, F_{4}\right)=1,
\end{aligned}
$$

hence

$$
\begin{aligned}
c_{3}(I)= & \operatorname{Vol}\left(v_{2}, F_{1}\right)+\min \left\{\operatorname{Vol}\left(v_{1}, F_{2}\right), \operatorname{Vol}\left(v_{2}, F_{2}\right)\right\}+ \\
& +\min \left\{\operatorname{Vol}\left(v_{1}, F_{3}\right), \operatorname{Vol}\left(v_{2}, F_{3}\right)\right\}+\operatorname{Vol}\left(v_{1}, F_{3}\right)= \\
= & 1+1+1+1=4 .
\end{aligned}
$$

We now present an example in dimension 5 in which several compact 1-dimensional faces lie on the same 3-unbounded facet. 
Example 7. Let $d=5, I=\left(x_{1}^{3} x_{2} x_{3} x_{4} x_{5}, x_{1} x_{2}^{2} x_{3} x_{4} x_{5}, x_{1} x_{2} x_{3} x_{4} x_{5}^{5}\right)$. By a computer computation we have

$$
\begin{aligned}
c(I)= & \left(c_{0}(I), c_{1}(I), c_{2}(I), c_{3}(I), c_{4}(I), c_{5}(I)\right)=(0,0,26,6,5,0)= \\
= & 22 \cdot(0,0,1,0,0,0)+2 \cdot(0,0,1,0,0,0)+2 \cdot(0,0,1,0,0,0)+ \\
& +2 \cdot(0,0,0,1,0,0)+(0,0,0,1,0,0)+(0,0,0,1,0,0)+ \\
& +(0,0,0,1,0,0)+(0,0,0,1,0,0)+(0,0,0,0,1,0)+ \\
& +(0,0,0,0,1,0)+(0,0,0,0,1,0)+(0,0,0,0,1,0)+ \\
& +(0,0,0,0,1,0),
\end{aligned}
$$

where the summands are the contributions of the highest dimensional components of the bigraded ring $T$, see Proposition 1.

The software Germenes [11] shows that the compact faces of $\Gamma(I)$ are the vertices $v_{1}=(3,1,1,1,1), v_{2}=(1,2,1,1,1), v_{3}=(1,1,1,1,5)$, the line segments $v_{1} v_{2}, v_{1} v_{3}, v_{2} v_{3}$ and the triangle $v_{1} v_{2} v_{3}$. The facets, all of them unbounded, are:

$$
\begin{aligned}
& F_{1}=v_{2} v_{3}+\mathbb{R}_{\geq 0} e_{2}+\mathbb{R}_{\geq 0} e_{3}+\mathbb{R}_{\geq 0} e_{4}+\mathbb{R}_{\geq 0} e_{5}, \\
& F_{2}=v_{1} v_{3}+\mathbb{R}_{\geq 0} e_{1}+\mathbb{R}_{\geq 0} e_{3}+\mathbb{R}_{\geq 0} e_{4}+\mathbb{R}_{\geq 0} e_{5}, \\
& F_{3}=v_{1} v_{2} v_{3}+\mathbb{R}_{\geq 0} e_{1}+\mathbb{R}_{\geq 0} e_{2}+\mathbb{R}_{\geq 0} e_{4}+\mathbb{R}_{\geq 0} e_{5}, \\
& F_{4}=v_{1} v_{2} v_{3}+\mathbb{R}_{\geq 0} e_{1}+\mathbb{R}_{\geq 0} e_{2}+\mathbb{R}_{\geq 0} e_{3}+\mathbb{R}_{\geq 0} e_{5}, \\
& F_{5}=v_{1} v_{2}+\mathbb{R}_{\geq 0} e_{1}+\mathbb{R}_{\geq 0} e_{2}+\mathbb{R}_{\geq 0} e_{3}+\mathbb{R}_{\geq 0} e_{4}, \\
& F_{6}=v_{1} v_{2} v_{3}+\mathbb{R}_{\geq 0} e_{3}+\mathbb{R}_{\geq 0} e_{4} .
\end{aligned}
$$

Obviously $\mathcal{F}(4)=\mathcal{F}(3)=\emptyset$, hence $c_{0}(I)=c_{1}(I)=0$.

We have $\mathcal{F}(2)=\left\{F_{3}, F_{4}, F_{6}\right\}$ and

$$
\begin{aligned}
\operatorname{Vol}\left(v_{1} v_{2} v_{3}, F_{3}\right) & =\operatorname{Vol}\left(v_{1} v_{2} v_{3}, F_{4}\right)=\min \left\{\left|\begin{array}{lll}
3 & 1 & 1 \\
1 & 2 & 1 \\
1 & 1 & 1
\end{array}\right|,\left|\begin{array}{lll}
1 & 1 & 1 \\
2 & 1 & 1 \\
1 & 1 & 5
\end{array}\right|\right\}= \\
& =\min \{2,4\}=2, \\
\operatorname{Vol}\left(v_{1} v_{2} v_{3}, F_{6}\right) & =\left|\begin{array}{lll}
3 & 1 & 1 \\
1 & 2 & 1 \\
1 & 1 & 5
\end{array}\right|=22 .
\end{aligned}
$$

Observe that in the computations of $\operatorname{Vol}\left(v_{1} v_{2} v_{3}, F_{3}\right)$ and $\operatorname{Vol}\left(v_{1} v_{2} v_{3}, F_{4}\right)$ four projections of the compact triangle $v_{1} v_{2} v_{3}$ give a line segment and 
must not be considered. Summing up we get

$$
\begin{aligned}
c_{2}(I) & =\operatorname{Vol}\left(v_{1} v_{2} v_{3}, F_{3}\right)+\operatorname{Vol}\left(v_{1} v_{2} v_{3}, F_{4}\right)+\operatorname{Vol}\left(v_{1} v_{2} v_{3}, F_{6}\right) \\
& =2+2+22=26 .
\end{aligned}
$$

The set of 3-unbounded facets containing 1-dimensional compact faces is $\mathcal{F}(1)=\left\{F_{1}, F_{2}, F_{3}, F_{4}, F_{5}\right\}$ and we have

$$
\begin{aligned}
& \operatorname{Vol}\left(v_{2} v_{3}, F_{1}\right)=\min \left\{\left|\begin{array}{ll}
1 & 1 \\
1 & 2
\end{array}\right|,\left|\begin{array}{ll}
1 & 1 \\
1 & 5
\end{array}\right|\right\}=\min \{1,4\}=1, \\
& \operatorname{Vol}\left(v_{1} v_{3}, F_{2}\right)=\min \left\{\left|\begin{array}{ll}
3 & 1 \\
1 & 1
\end{array}\right|,\left|\begin{array}{ll}
1 & 1 \\
1 & 5
\end{array}\right|\right\}=\min \{2,4\}=2, \\
& \operatorname{Vol}\left(v_{1} v_{2}, F_{3}\right)=\operatorname{Vol}\left(v_{1} v_{2}, F_{4}\right)=\min \left\{\left|\begin{array}{ll}
3 & 1 \\
1 & 1
\end{array}\right|,\left|\begin{array}{ll}
2 & 1 \\
1 & 1
\end{array}\right|\right\}=\min \{2,1\}=1, \\
& \operatorname{Vol}\left(v_{1} v_{3}, F_{3}\right)=\operatorname{Vol}\left(v_{1} v_{3}, F_{4}\right)=\min \left\{\left|\begin{array}{ll}
3 & 1 \\
1 & 1
\end{array}\right|,\left|\begin{array}{ll}
1 & 1 \\
1 & 5
\end{array}\right|\right\}=\min \{2,4\}=2, \\
& \operatorname{Vol}\left(v_{2} v_{3}, F_{3}\right)=\operatorname{Vol}\left(v_{2} v_{3}, F_{4}\right)=\min \left\{\left|\begin{array}{ll}
2 & 1 \\
1 & 1
\end{array}\right|,\left|\begin{array}{ll}
1 & 1 \\
1 & 5
\end{array}\right|\right\}=\min \{1,4\}=1, \\
& \operatorname{Vol}\left(v_{1} v_{2}, F_{5}\right)=\min \left\{\left|\begin{array}{ll}
3 & 1 \\
1 & 1
\end{array}\right|,\left|\begin{array}{ll}
2 & 1 \\
1 & 1
\end{array}\right|\right\}=\min \{2,1\}=1,
\end{aligned}
$$

hence

$$
\begin{aligned}
c_{3}(I)= & \operatorname{Vol}\left(v_{2} v_{3}, F_{1}\right)+\operatorname{Vol}\left(v_{1} v_{3}, F_{2}\right)+ \\
& +\min \left\{\operatorname{Vol}\left(v_{1} v_{2}, F_{3}\right), \operatorname{Vol}\left(v_{1} v_{3}, F_{3}\right), \operatorname{Vol}\left(v_{2} v_{3}, F_{3}\right)\right\}+ \\
& +\min \left\{\operatorname{Vol}\left(v_{1} v_{2}, F_{4}\right), \operatorname{Vol}\left(v_{1} v_{3}, F_{4}\right), \operatorname{Vol}\left(v_{2} v_{3}, F_{4}\right)\right\}+ \\
& +\operatorname{Vol}\left(v_{1} v_{2}, F_{5}\right)=1+2+1+1+1=6 .
\end{aligned}
$$

From the list of the facets we see that there are five 4-unbounded facets, precisely $\mathcal{F}(0)=\left\{F_{1}, F_{2}, F_{3}, F_{4}, F_{5}\right\}$ and we have

$$
\begin{aligned}
& \operatorname{Vol}\left(v_{2}, F_{1}\right)=1, \quad \operatorname{Vol}\left(v_{3}, F_{1}\right)=1, \quad \operatorname{Vol}\left(v_{1}, F_{2}\right)=1, \quad \operatorname{Vol}\left(v_{3}, F_{2}\right)=1, \\
& \operatorname{Vol}\left(v_{1}, F_{3}\right)=1, \quad \operatorname{Vol}\left(v_{2}, F_{3}\right)=1, \quad \operatorname{Vol}\left(v_{3}, F_{3}\right)=1, \quad \operatorname{Vol}\left(v_{1}, F_{4}\right)=1, \\
& \operatorname{Vol}\left(v_{2}, F_{4}\right)=1, \quad \operatorname{Vol}\left(v_{3}, F_{4}\right)=1, \quad \operatorname{Vol}\left(v_{1}, F_{5}\right)=1, \quad \operatorname{Vol}\left(v_{2}, F_{5}\right)=1, \\
& c_{4}(I)=\min \left\{\operatorname{Vol}\left(v_{2}, F_{1}\right), \operatorname{Vol}\left(v_{3}, F_{1}\right)\right\}+\min \left\{\operatorname{Vol}\left(v_{1}, F_{2}\right), \operatorname{Vol}\left(v_{3}, F_{2}\right)\right\}+ \\
& +\min \left\{\operatorname{Vol}\left(v_{1}, F_{3}\right), \operatorname{Vol}\left(v_{2}, F_{3}\right), \operatorname{Vol}\left(v_{3}, F_{3}\right)\right\}+ \\
& +\min \left\{\operatorname{Vol}\left(v_{1}, F_{4}\right), \operatorname{Vol}\left(v_{2}, F_{4}\right), \operatorname{Vol}\left(v_{3}, F_{4}\right)\right\}+ \\
& +\min \left\{\operatorname{Vol}\left(v_{1}, F_{5}\right), \operatorname{Vol}\left(v_{2}, F_{5}\right)\right\}=1+1+1+1+1=5 .
\end{aligned}
$$




\section{REFERENCES}

[1] R. Achilles and D. Aliffi, Segre, a script for the Reduce package Cali, Bologna, 1999-2017, http://www.dm.unibo.it/ ${ }^{\sim}$ achilles/segre/.

[2] R. Achilles and M. Manaresi, Multiplicity for ideals of maximal analytic spread and intersection theory, J. Math. Kyoto Univ. 33 (1993), 569-578.

[3] R. Achilles and M. Manaresi, Multiplicities of a bigraded ring and intersection theory, Math. Ann. 309 (1997), 573-591.

[4] R. Achilles and S. Rams, Intersection numbers, Segre numbers and generalized Samuel multiplicities, Arch. Math. (Basel) 77 (2001), 391-398.

[5] C. Bivià-Ausina, The analytic spread of monomial ideals, Comm. Algebra 31 (2003), No. 7, 3487-3496

[6] D. Eisenbud and B. Sturmfels, Binomial ideals, Duke Math. J. 84 (1996), 145.

[7] G. Guibert, Classe de Segre locale d'un idéal et frontières de Newton, C. R. Acad. Sci. Paris Sér. I Math. 329, (1999), 315-320.

[8] A. C. Hearn, ReducE: A user-oriented interactive system for algebraic simplification. In M. Klerer and J. Reinfelds, editors, Interactive Systems for Experimental Applied Mathematics, pages 79-90, New York, 1968. Academic Press.

[9] J. Jeffries and J. Montaño, The j-multiplicity of monomial ideals, Math. Res. Lett. 20 (2013), No. 4, 729-744.

[10] R. Kummer and B. Renschuch, Potenzproduktideale. I., Publ. Math. Debrecen 17 (1970), 81-98 (1971).

[11] A. Montesinos, Germenes, program available at http://www.uv.es/montesin/.

[12] Reduce: A portable general-purpose computer algebra system, https://reduce-algebra.sourceforge.io/

[13] L. Reid and M. A. Vitulli, The weak subintegral closure of a monomial ideal, Comm. Algebra 27 (1999), 5649-5667.

[14] B. Teissier, Monômes, volumes et multiplicités, Introduction à la théorie des singularités, II, 127-141, Travaux en Cours, 37, Hermann, Paris, 1988.

[15] B. L. van der Waerden, On Hilbert's function, series of composition of ideals and a generalisation of the theorem of Bezout, Proc. Roy. Acad. Amsterdam 31 (1929), 749-770.

Dipartimento di Matematica, Università di Bologna, Piazza di Porta

S. Donato 5, 40126 Bologna, Italy

E-mail address: rudiger.achilles@unibo.it, mirella.manaresi@unibo.it 